Les ANNALES Les Annales de droit

DE DROIT

12 | 2018

Varia

\title{
La souveraineté et la norme fondamentale
}

Sovereignty and the fundamental norm

Boris Barraud

\section{(2) OpenEdition}

Journals

Édition électronique

URL : http://journals.openedition.org/add/878

DOI : $10.4000 /$ add. 878

ISSN : 2606-1988

Éditeur

Presses universitaires de Rouen et du Havre

Édition imprimée

Pagination : 09-30

ISBN : 979-10-240-0937-7

ISSN : 1955-0855

\section{Référence électronique}

Boris Barraud, «La souveraineté et la norme fondamentale », Les Annales de droit [En ligne], 12 | 2018, mis en ligne le 05 février 2019, consulté le 05 décembre 2019. URL : http://journals.openedition.org/ add/878; DOI : 10.4000/add.878

Presses universitaires de Rouen et du Havre 


\section{La souveraineté et la norme fondamentale}

Boris BARRAUD

«Le problème du fondement du droit ne fait qu'un avec le problème du fondement de l'État", écrivait Raymond Carré de Malberg ${ }^{1}$. Ne faut-il pas, dès lors, considérer que le problème de la norme fondamentale ne ferait qu'un avec le problème de la souveraineté ? Telle est la problématique développée en ces pages, étant précisé que la norme fondamentale, en tant que supposition à des fins logiques, doit s'analyser telle une théorie et non telle une norme en bonne et due forme, non telle une norme effective, si bien qu'il s'agit ici de rapprocher deux théories ou, mieux, deux concepts et non un concept et une règle de droit positif.

Classiquement, les professeurs de droit public ou de science politique enseignent à leurs étudiants que, pour comprendre ce qu'est l'État, il faudrait déjà comprendre ce qu'est la souveraineté, que cette dernière serait l'élément essentiel de la théorie de l'État. Par ailleurs, nombre de théoriciens du droit contemporains, influencés par l'héritage kelsénien, soutiennent que les règles de droit seraient ordonnées les unes par rapport aux autres à travers des rapports de validité et qu'au sommet de la «pyramide» que les normes formeraient, il se trouverait une norme fondamentale, norme hypothétique ou supposée à des fins logiques et non positive, ne requérant pas d'être elle-même validée par quelque norme supérieure et permettant de justifier la pleine validité de la constitution historiquement première et de toutes les règles y faisant suite. La thèse que cette étude souhaite proposer et défendre est la suivante: les uns et les autres parleraient en réalité de la même chose, «souveraineté » étant le terme propre à la théorie de l'État servant à désigner ce à quoi «norme fondamentale» renvoie dans la théorie du droit.

Ensuite, en raison du caractère fortement controversable, indéterminé et mouvant de la souveraineté, là où le sens de l'expression «norme

1. Raymond Carré de Malberg, Contribution à la théorie générale de l'État spécialement d'après les données fournies par le droit constitutionnel français, Paris, Recueil Sirey, 1920 , t. I, p. 67. 
fondamentale» est difficilement discutable tant celle-ci s'attache à un auteur et à une œuvre précisément identifiés, la souveraineté est appelée à se rapprocher de la norme fondamentale bien plus que la norme fondamentale n'est appelée à se rapprocher de la souveraineté. Il semble davantage porteur de définir la souveraineté en tant que norme fondamentale que de définir la norme fondamentale en tant que souveraineté.

Les penseurs de l'État justifient son monopole du droit par la souveraineté, tandis que les penseurs du droit expliquent l'exacte superposition des dimensions de l'État et des dimensions du droit par la norme fondamentale, mère idéelle des normes juridiques. Pour les normativistes selon lesquels État et droit ne feraient qu'un, seraient deux mots différents servant à désigner un même objet, il paraît difficile, dès lors qu'est posée la question de la souveraineté, de ne pas y voir une autre façon de désigner la norme fondamentale. Mais l'intimité entre souveraineté et norme fondamentale n'est-elle pas également grande parmi les discours des non-normativistes, qui distinguent droit et État, pensée du droit et pensée de l'État? Néanmoins, un tel rapprochement confinant à la confusion de la souveraineté et de la norme fondamentale n'a, semblet-il, jamais été envisagé ou, du moins, jamais été soutenu explicitement.

La définition de la souveraineté ici retenue est la suivante: elle serait une norme suprême fictive servant à faire accepter la puissance juridique de l'État, la source d'une métaprérogative conférant à l'État l'indépendance juridique vis-à-vis de l'extérieur et la suprématie et l'omnicompétence juridiques à l'intérieur. La souveraineté serait une norme (supposée et non positive, donc un concept) attributive de compétence conférant à l'État un droit dont il serait le seul titulaire originaire possible: le droit au droit. Grâce à cette métaprérogative juridique, l'État pourrait commander et dominer légitimement et totalement du seul fait qu'il est État. En d'autres termes, la souveraineté désignerait la norme fondamentale de l'ordre juridique. Il en résulte que la caractéristique spécifique et le signe distinctif de l'État seraient également à rechercher dans cette norme fondamentale, laquelle se retrouverait par conséquent projetée au cœur de la théorie juridique de l'État.

Au moment d'édifier l'État moderne, Jean Bodin et ses successeurs ont entendu concentrer dans les mains du monarque diverses attributions indispensables au gouvernement du royaume. En particulier, il fallait investir le «souverain » de la maîtrise de la force juridique, laquelle est une force symbolique et psychologique avant d'être une force matérielle. C'est ainsi qu'à partir du $\mathrm{XVI}^{\mathrm{e}}$ siècle se sont développés concomitamment les souverainetés étatiques et les ordres juridiques nationaux, ces derniers procédant de ces premières. Aujourd'hui, on affirme que l'État 
souverain dispose de la "compétence de la compétence», c'est-à-dire du «droit au droit», d'un entier contrôle du droit applicable sur son territoire et à l'égard de sa population, et que telle est la portée de la souveraineté. In fine, tout acte juridique, quelle que soit la forme qu'il prenne, serait une application de la souveraineté étatique. Il est alors tentant de rapprocher la souveraineté de la norme fondamentale. Cela implique évidemment d'accepter la théorie normativiste kelsénienne, et en premier lieu la thèse de la norme fondamentale. Or, dans la théorie juridique de l'État de Kelsen, les institutions étatiques se réduisent à une "pyramide» de normes, de telle sorte que, si l'on rapproche la souveraineté du droit, on la rapproche nécessairement de la norme fondamentale qui chapeaute cette "pyramide ».

On s'intéresse actuellement moins à la signification et à la portée de la souveraineté - alors pourtant que cette notion s'avère excessivement polysémique - qu'aux problèmes de son effacement, de son dépassement et de sa dissolution ${ }^{2}$. Quant à la fiction de la norme fondamentale, si les questions de sa logique, de sa pertinence et de son utilité ont agité la doctrine jus théorique durant la seconde moitié $\mathrm{du} \mathrm{xx}^{\mathrm{e}}$ siècle, il en va désormais différemment et nombreux sont les théoriciens du droit positivistes qui ne s'attardent guère sur la problématique de la validité des normes historiquement et hiérarchiquement premières de l'ordre juridique. Néanmoins, face à la rareté, à l'ère contemporaine, des recherches portant sur la souveraineté ou sur la norme fondamentale et face à l'absence de travaux portant sur la souveraineté et sur la norme fondamentale, il paraît opportun d'entreprendre une étude consacrée à la souveraineté-norme fondamentale. La souveraineté semble bien devoir demeurer, au moins encore pour quelque temps, une notion indépassable; et, si elle "n'a rien d'évident ni de spontané ${ }^{3}$ ", si elle pèche par manque de clarté et de fermeté sémantiques, il faut croire qu'il n'est pas inconsidéré de vouloir contribuer à son éclaircissement et à son affermissement sémantiques en remettant au premier plan des

2. Par exemple, Pauline Türk, «La souveraineté des États à l'épreuve d'internet», RDP 2013, p. 1489 et suiv.; Félicien Lemaire, «Propos sur la notion de "souveraineté partagée" ou sur l'apparence de remise en cause du paradigme de la souveraineté », RFD const. 2012, p. 821 et suiv.; Jim Lapin, "L'équilibre des finances publiques: une exigence externe mettant en cause la souveraineté de l'État», RDP 2014, p. 733 et suiv.; Pierre Pescatore, "La souveraineté dans une société d'inégaux, pouvoir suprême... coalisable, partageable, divisible, intégrable... responsable ? justiciable ?", dans Mélanges Jean-Pierre Puissochet, Paris, Pedone, 2008, p. 231 et suiv.

3. François de Smet, Le mythe de la souveraineté. Du Corps au Contrat social, Paris, EME, 2014, p. 7. 
enjeux s'y attachant celui de sa définition. Tel est le principal objectif des présentes pages : contribuer à l'éclaircissement du sens et de la portée de ce terme omniprésent, mais obscur - omniprésent, car obscur? - qu'est «souveraineté».

Certainement la souveraineté a dû affronter les critiques de quelques détracteurs ${ }^{4}$. Ces approches négatives n'ont rencontré qu'un succès modeste et aujourd'hui on va jusqu'à faire de l'opinion selon laquelle la souveraineté serait l'élément cardinal de la pensée de l'État non pas une opinion devenue majoritaire, mais une opinion devenue unanime ${ }^{5}$. Il faut croire que plus aucun théoricien de l'État ne se risquerait à le définir sans recourir à la souveraineté. Seulement, après avoir défini l'État telle une personne souveraine, encore faut-il comprendre ce que recouvre le qualificatif «souveraine». Si la souveraineté caractérise et donc définit l'État, qu'est-ce qui caractérise et définit la souveraineté? Et, en premier lieu, s'agit-il d'une notion de juristes, d'une notion de politistes, d'une notion de philosophes? Il est délicat de répondre, car le sens de la souveraineté est ambivalent, mal situé, relativement flou. C'est pourquoi la qualité juridique de la souveraineté est, dans le contexte doctrinal actuel, une possibilité, mais pas une nécessité, alors que la juridicité de la norme fondamentale ne fait aucun doute - quoique soit en cause une juridicité logique, mais fictive, non une juridicité positive. La rencontre de la souveraineté et du droit peut être qualifiée de "contingente » puisque ladite souveraineté a été initialement pensée telle une "puissance absolue et perpétuelle», soit telle une force factuelle ou telle une onction théologique, mais non telle une norme juridique ${ }^{6}$. Cependant, la signification de la souveraineté a évolué et cette évolution a tendu à toujours plus la rapprocher du droit, si bien qu'aujourd'hui, si

4. Notamment, Léon Duguit ne voyait en elle qu'un outil symbolique au service de la domination politique et «aboutiss[ant] forcément à la négation du droit public, [...] à l'absolutisme et au despotisme" (Traité de droit constitutionnel, t. II: La théorie générale de l'État, $1^{\text {re }}$ partie : Éléments, fonctions et organes de l'État, Paris, De Boccard, 1928, p. 117); tandis que Benjamin Constant se proposait de «détruire la notion de souveraineté », car «c'est contre l'arme et non contre le bras qu'il faut sévir». Suivant cette logique, il cherchait à démontrer que «le courroux des hommes s'est dirigé contre les possesseurs du pouvoir et non contre le pouvoir lui-même». " $\mathrm{Au}$ lieu de détruire ce pouvoir, ils n'ont songé qu’à le déplacer [du roi vers la nation]. C'était un fléau, ils l'ont considéré comme une conquête». (Principes de politique, Paris, Guillaumin, 1872, p. 10).

5. Éric Maulin, «Souveraineté», dans Denis Alland et Stéphane Rials (dir.), Dictionnaire de la culture juridique, Paris, PUF, 2003, p. 1438.

6. Thomas Berns, "Souveraineté, droit et gouvernementalité », Arch. phil. droit 2002, p. 360 ; Jean Bodin, Les six livres de la République, 1576, L. I, chap. 8. 
une définition dominante se dégage des essais de théorie de l'État et des manuels de droit constitutionnel ou de science politique, cette définition s'avère largement d'ordre juridique ${ }^{7}$.

Or, aux yeux du théoricien du droit, la souveraineté fait fatalement penser à la norme fondamentale, à tel point qu'il est tentant de considérer qu'elle ne désignerait rien d'autre que cette norme fondamentale. La souveraineté a néanmoins toujours été pensée et étudiée indépendamment de la norme fondamentale - et réciproquement -, ce qu'il importe de remarquer (1) avant de proposer de les relier (2).

\section{Les trajectoires séparées de la souveraineté et de la norme fondamentale}

Avant d'envisager une unitaire souveraineté-norme fondamentale, il convient tout d'abord de souligner combien il est habituel, non pas d'opposer la souveraineté et la norme fondamentale, mais de les aborder comme si elles étaient des notions indifférentes l'une à l'autre, comme si elles s'attachaient à des champs et à des portées incomparables. Il ne semble ainsi guère exister de travaux interrogeant tout à la fois la souveraineté et la norme fondamentale. C'est en premier lieu ce constat qui a conduit aux réflexions exposées en ces pages. Il est vrai que souveraineté et norme fondamentale présentent des caractéristiques doctrinales très dissemblables: la première, bien que son sens soit incertain, rencontre un grand succès (1.1) quand la seconde, dont le sens est précisément fixé, est d'emploi beaucoup moins courant et souvent critiquée (1.2) - en réalité, il existe certainement un rapport de cause à effet entre succès doctrinal et imprécision sémantique. Par la suite, la polysémie qui marque la souveraineté, là où la norme fondamentale est à l'inverse monosémique, amène à les considérer comme synonymes en rapprochant la souveraineté de la norme fondamentale plutôt qu'en rapprochant la norme fondamentale de la souveraineté. C'est bien plus la signification de la souveraineté que celle de la norme fondamentale qu'il s'agit ici de discuter.

7. Il est de mieux en mieux admis que l'État est une « construction auxiliaire de la pensée juridique» (Hans Kelsen, Théorie pure du droit, C. Eisenmann (trad.), Paris, Dalloz, 1962, p. 387). 


\subsection{Le sens imprécis, mais incontesté de la souveraineté}

La paternité de la souveraineté moderne - et, par la même occasion, de l'État moderne - peut être attribuée à Jean Bodin ${ }^{8}$ qui, au Xvi ${ }^{\mathrm{e}}$ siècle, a entendu consolider l'autorité de la « République » en en faisant la titulaire d'une "puissance absolue et perpétuelle», en la concevant tel un «droit gouvernement de plusieurs mesnages [...] avec puissance souveraine ${ }^{9}$ » - mais en soutenant tout de même, comme Thomas Hobbes, que cette puissance serait une "faveur de Dieu ${ }^{10}$ " obligeant à «respecter le modèle de la loi divine». Le jurisconsulte angevin notait, par exemple, que "seul le titre de souveraineté est le vray fondement et le pivot sur lequel tourne l'estat d'une société, et de laquelle dépendent tous les magistrats, loix et ordonnances ${ }^{11} »$. La souveraineté revêt donc un caractère nettement plus ancien que la norme fondamentale, création kelsénienne du $\mathrm{xx}^{\mathrm{e}}$ siècle.

Aujourd'hui, on enseigne communément que, "pour comprendre ce qu'est l'État, il faut analyser une autre notion: celle de souveraineté ${ }^{12}$ ». La difficulté est que le sens de cette souveraineté est controversé et équivoque. Au début du $\mathrm{xx}^{\mathrm{e}}$ siècle, Carré de Malberg, après avoir passé en revue les multiples signifiés qui étaient alors attachés au mot "souveraineté", en concluait qu'il ne pouvait guère s'agir que d'un "concept embrouillé et obscur», ayant subi d'excessives extensions ${ }^{13}$. Un même constat peut sans doute être opéré actuellement et d'aucuns en viennent à qualifier la souveraineté de «concept aussi inconsistant qu'incontournable», de "concept non élucidé» entrânant le «malaise des juristes à son égard» ou encore de "concept frustrant», car mêlant "pauvreté et suffisance ${ }^{14}$ ». L'idée de souveraineté est employée afin de désigner de nombreuses situations politiques ou juridiques relatives soit à la légitimité du pouvoir, soit à l'exercice de certaines prérogatives, soit

8. Voir Olivier Beaud, La puissance de l'État, Paris, PUF, 1994, p. 30 et 52 ; Gérard Mairet, Le principe de souveraineté. Histoire et fondements du pouvoir moderne, Paris, Gallimard, 1997.

9. Jean Bodin, Les six livres de la République, 1576, L. I, chap. 8 et L. I, chap. 1.

10. Thomas Hobbes, Léviathan, 1651, chap. 23.

11. Jean Bodin, Les six livres de la République, 1576, L. I, chap. 8 et L. I, chap. 2.

12. Denis Baranger, Droit constitutionnel, Paris, PUF, 2013, p. 35.

13. Raymond Carré de Malberg, Contribution à la théorie générale de l'État..., op. cit., t. I, p. 79.

14. Thomas Berns, "Souveraineté, droit et gouvernementalité», art. cité, p. 353; François de Smet, Aux origines théologiques de la souveraineté, Paris, EME, 2015; Thomas Berns, Souveraineté, droit et gouvernementalité. Lectures du politique moderne à partir de Bodin, Paris, Léo Scheer, 2005, p. 1. 
à la définition de l'État ${ }^{15}$. Sont évoquées aussi bien la souveraineté de l'État que celles de certains organes ou même de certains gouvernants. Notamment, le roi, la nation, le peuple, le législateur et le constituant seraient tour à tour souverains. On retient souvent que le Conseil d'État et la Cour de cassation, parce qu'ils décident à titre définitif et irrévocable et que leurs arrêts sont insusceptibles de tout recours, seraient des " cours souveraines». Jusqu'à des objets intrinsèquement ni juridiques ni politiques, tels que l'internet, pourraient être souverains ${ }^{16}$.

La souveraineté connaît donc des significations multiples, si bien que, comme tous les termes qui revêtent trop de sens, elle risque de finir par ne plus en revêtir aucun. La "crise de la souveraineté », souvent évoquée à l'ère de la mondialisation du droit, à l'heure du droit international et du droit transnational, semble être en réalité autant une crise théorique de la notion qu'une crise concrète de ses effets ${ }^{17}$. En tout cas, tout travail de recherche entendant mobiliser la souveraineté devrait au préalable expliquer quelle est la conception qu'il en retient.

L'évocation de la souveraineté est également rendue périlleuse par le fait qu'elle transcende les frontières académiques, qu'elle se situe à la croisée des chemins du droit, de la politique et de la philosophie. De plus, à l'extérieur des facultés de droit et de science politique, « souveraineté » est aussi un mot très usité dans le monde de l'action et du commentaire politique. Il compte parmi ces quelques termes d'essence juridico-politique allègrement entrés dans le langage courant, à tel point que chacun, du journaliste au simple citoyen, l'utilise fréquemment et sans grandes précautions. Son succès a contribué à en faire un mot excessivement polysémique et, par conséquent, trop peu précis pour pouvoir appartenir a priori à un langage scientifique. La souveraineté est donc problématique à plus d'un titre et un auteur peut expliquer que le «sentiment de "malaise" d'une modernité en quête de repères consensuels » serait à imputer à la facilité que constitue le fait d'accepter des notions comme "souveraineté" comme des données atomiques dont le sens et la portée seraient universellement évidents et compris, et sur lesquels le consensus social aurait été depuis longtemps établi ». «Rien n'est plus douteux pourtant: la souveraineté est un voile, et il nous faut comprendre ce qu'elle cache ${ }^{18}$. Or, depuis le milieu du $\mathrm{Xx}^{\mathrm{e}}$ siècle, il n’y

15. Éric Maulin, «Souveraineté», art. cité, p. 1434.

16. Par exemple, Karim Benyekhlef, «L'internet: un reflet de la concurrence des souverainetés", Lex Electronica, vol. 8, 2002, p. 1 et suiv.

17. Voir Bernard Cubertafond, «Souverainetés en crise?», RDP 1989, p. 1273 et suiv.

18. François de Smet, Le mythe de la souveraineté..., op. cit., p. 8. 
a pratiquement plus eu, en France, de véritables discussions doctrinales autour de la souveraineté ni de grandes entreprises théoriques la prenant comme objet ${ }^{19}$ - un sort particulier devant néanmoins être réservé à La puissance de l'État d'Olivier Beaud.

Ensuite, la souveraineté se présentant telle la notion cardinale, tel l'élément clef de la pensée de l'État, les incertitudes qui l'entourent rejaillissent directement sur lui. Il faut croire que les constituants français ne s'y sont pas trompés puisque le mot et ses dérivés lexicaux ne se retrouvent qu'à trois reprises dans le texte de la Constitution du 4 octobre 1958 ; et est évoquée la "souveraineté nationale», non la souveraineté de l'État ${ }^{20}$. Même si la souveraineté intègre ainsi l'«identité constitutionnelle nationale ${ }^{21}$ ", elle appartient avant tout à la doctrine, aux penseurs de l'État et de la chose publique ; et ceux-ci ne sont pas parvenus à en faire un objet précisément identifié et consensuellement accepté. Georges Burdeau allait jusqu'à voir dans la souveraineté la «question la plus épineuse du droit public ${ }^{22} »$. Reste que cette souveraineté est profondément ancrée dans la psyché juridico-politique collective, à tel point qu'elle semble être devenue «mythique» aux yeux de la plupart des théoriciens de l'État. Certainement Léon Duguit l'a vigoureusement dénoncée, l'envisageant telle une "métaphysique sans valeur ${ }^{23}$ ", mais rares sont ceux qui ont suivi l'illustre professeur bordelais dans cette voie et, à l'heure actuelle, il ne se trouve guère d'ouvrages consacrés à l'État ne plaçant pas la souveraineté au cœur des démonstrations et des réflexions.

En ces lignes, la conception de la souveraineté retenue l'attache à l'État seul, ce qui interdit d'envisager quelque souveraineté d'une haute juridiction, du constituant, de la nation ou du peuple. La souveraineté est alors l'élément définitionnel décisif de l'État. Si ce dernier se conçoit telle une personne souveraine, il partage la personnalité morale avec des entités qui ne sont pas des États, mais ne partage la souveraineté avec personne. Bien sûr, il s'agit là d'un choix et il n'est pas lieu d'ignorer que, tant historiquement qu'actuellement, souveraineté et État ne sont pas nécessairement liés ${ }^{24}$. La définition de l'État en tant que personne souveraine est toutefois celle agréée de Raymond Carré de Malberg à

19. Olivier Beaud, «Préface», dans Carl Schmitt, Théorie de la Constitution (1928), Paris, PUF, 1992.

20. Const. 4 oct. 1958 , préambule, art. 3 et 4 .

21. Bruno de Witte, "Droit communautaire et valeurs constitutionnelles nationales", Droits, $\mathrm{n}^{\mathrm{o}} 14,1991$, p. 90.

22. Georges Burdeau, L'État (1970), Paris, Seuil, 2009, p. 64.

23. Léon Duguit, Traité de droit constitutionnel..., op. cit., p. 1.

24. Jacques Baguenard, L'État: une aventure incertaine, Paris, Ellipses, 1998, p. 64. 
Georges Vedel en passant par nombre des juristes publicistes contemporains ${ }^{25}$.

La souveraineté est ici conçue telle la prérogative juridique suprême, le «droit propre et originel de domination» que décrivait Paul Laband ${ }^{26}$. À nouveau, cela n'est qu'une option parmi d'autres et il faut rappeler combien il existe des conceptions ajuridiques, politiques en premier lieu, de la souveraineté. Avant de développer davantage cette approche de la souveraineté, qui consiste à la connecter à la norme fondamentale et même à la confondre avec, il faut revenir sur cette dernière. La norme fondamentale contraste avec la souveraineté, car sa signification et sa portée ne font guère de doutes alors que son succès est très mitigé puisqu'il s'agit peut-être de la proposition kelsénienne qui a été la plus largement contestée, si ce n'est rejetée, par les théoriciens du droit positivistes.

\subsection{Le sens précis, mais contesté de la norme fondamentale}

Pour qu'un certain ensemble de normes constitue un ordre juridique et non un simple agrégat de normes disparates, il est nécessaire qu'existe un principe unificateur qui organise ces normes grâce à un certain type de relations leur permettant de se structurer, chaque norme trouvant sa place vis-à-vis des autres. Ce principe, qui est aussi le principe du normativisme, est que "la validité d'une norme ne peut avoir d'autre fondement que la validité d'une autre norme ${ }^{27}$ ». La validité est l'inclusion au sein d'un ordre juridique; elle est, d'un point de vue normativiste, le «mode d'existence spécifique des normes [juridiques]». Sous un angle statique ou matériel, une norme est valide dès lors qu'elle respecte le contenu des normes supérieures; sous un angle dynamique ou formel, une norme est valide dès lors qu'elle a été posée conformément aux prescriptions procédurales établies par les normes supérieures. Les normes sont ainsi hiérarchisées et forment un ordre juridique qu'il est permis de figurer sous les traits d'une "pyramide» puisque plus les normes sont haut placées dans la hiérarchie, moins elles sont nombreuses.

25. Raymond Carré de Malberg, Contribution à la théorie générale de l'État..., op. cit., t. I, p. 9; Georges Vedel, Droit constitutionnel, Paris, Recueil Sirey, 1949, p. 99 ( l'État se définit comme une personne morale munie de la souveraineté »).

26. Paul Laband, Le droit public de l'Empire allemand, t. I, C. Gandilhon (trad.), Paris, Giard et Bière, 1900 (cité par Jacky Hummel, «Allemagne (doctrines allemandes de l'État et du droit de Hegel et Jellinek) », dans Dictionnaire de la culture juridique, op. cit., p. 43).

27. Hans Kelsen, Théorie pure du droit, op. cit., p. 256. 
Au sommet de cette "pyramide » des normes positives se trouvent les normes portées par la constitution. Par définition, celles-ci ne sont validées par aucune norme positive supérieure - il est permis de remonter jusqu'à la constitution historiquement première, mais le problème est alors seulement transporté à son niveau. Il faut pourtant, d'une manière ou d'une autre, juger valides ces normes positives supérieures, sans quoi leur invalidité rejaillirait sur l'ensemble de l'ordre normatif. La théorie kelsénienne retient qu'il faut simplement «supposer la validité de la Constitution $^{28}$ ». Et, afin de faciliter cette supposition et l'établissement de la validité objective de l'ordre juridique, est postulée l'existence d'une norme supérieure aux règles d'essence constitutionnelle, qui leur confère la validité sans avoir besoin d'être elle-même validée, car elle est la "norme fondamentale» (Grundnorm en allemand, Basic Norm en anglais) de l'ordre juridique. De cette façon, le fait peut être tenu loin du droit et la rupture humienne entre être et devoir-être peut être entièrement préservée. L'enjeu est, pour la « théorie pure du droit », de pouvoir tenir pour droit positif un système normatif sans devoir recourir à des considérations factuelles, historiques ou politiques telles que l'effectivité des normes ou l'avènement de la constitution historiquement première.

La norme fondamentale, premier domino normatif, est une métaphore à visée explicative et logique - à l'instar, par exemple, du « contrat social»-, non une norme réelle et positive; elle est supposée et non posée, pensée et non voulue, le résultat d'un acte de connaissance et non celui d'un acte de volonté ${ }^{29}$. C'est pour cela qu'il n'est pas nécessaire qu'elle soit elle-même validée par une norme supérieure. Pour Kelsen, elle n'est pas un postulat arbitraire, mais quelque chose qui s'impose à la raison comme une conséquence immédiate et inévitable. Afin d'éviter que le système de validation des règles de droit ne se perde dans une peu commode regressio infinitum, il lui est apparu indispensable de postuler l'existence de quelque «norme dernière et suprême » étant la «source commune de la validité de toutes les normes qui appartiennent à l'ordre juridique». Il s'agit, selon Kelsen, d'une «hypothèse logique transcendantale ${ }^{30} »$; mais est en cause, plus sûrement - car une hypothèse doit pouvoir être vérifiée -, une fiction, une supposition, une condition logique servant une démarche scientifique, « un expédient de

28. Ibid., p. 13, 258 et suiv., et 265.

29. Henri Thévenaz, "Le théorème de Gödel et la norme fondamentale de Kelsen", Dr. et société 1986, p. 533 ; Michel Troper, «La pyramide est toujours debout", RDP, 1978, p. 1526, Hans Kelsen, Théorie pure du droit, op. cit., p. 269 et 272.

30. Ibid., p. 268,257 et 269 . 
la pensée dont on se sert si l'on ne peut atteindre le but de la pensée avec les données dont on dispose ${ }^{31} »$.

Ensuite, le théoricien du droit austro-américain a imaginé un contenu pour la norme fondamentale: "On doit se conduire de la façon que la constitution prescrit. " Mais l'important, en ces lignes, est que la norme fondamentale soit ce qui assure aux normes portées par la constitution actuelle et par la constitution historiquement première d'être valides et donc juridiques. Ainsi, elle consacre le droit au droit de l'État, prérogative juridique suprême. Elle semble être, davantage qu'une norme prescriptive, une norme attributive de compétence, conférant à l'État le pouvoir d'édicter et d'appliquer les règles de droit.

Kelsen expliquait par ailleurs qu'il ne saurait y avoir d'ordre juridique qu'à condition que les règles qui le composent soient "effectivement obéies et appliquées en gros et de façon générale ${ }^{32} »$. Peut-être ne lui aurait-il pas fallu chercher ailleurs la justification de la validité des normes de cet ordre juridique et, en premier lieu, des normes à valeur constitutionnelle, quitte à abandonner l'idéal d'autospécification du droit; mais le principe même du normativisme l'interdit, interdit de faire reposer l'ordre juridique sur la révolution, le consensus politique, la volonté du peuple, l'emprise du tyran ou la force policière ou armée: en aucune façon des faits ne peuvent expliquer pourquoi on qualifie certaines choses de «juridiques». L'État et le droit ne sauraient être les conséquences d'une violence sans fondement, d'un coup de force ou d'un "coup d'écriture ${ }^{33}$ " puisque le monde du droit et ses liens d'imputation ne sauraient être mêlés au monde du fait et à ses liens de causalité. La fin de la norme fondamentale est bien de garantir l'autonomie du droit.

Néanmoins, l'utilité de la fiction de la norme fondamentale est douteuse $^{34}$. Elle explicite un présupposé qui gagnerait peut-être à demeurer dans l'espace du non-dit. Elle se présente en tout cas tel le point le plus problématique de la «théorie pure du droit». Expression d'un

31. Michel Troper, «Normativisme», dans Dictionnaire de la culture juridique, op. cit., p. 1076; Stanley S. Paulson, «Hans Kelsen et les fictions juridiques », Droits, $\mathrm{n}^{\circ} 21$, 1995, p. 75 et suiv. ; Éric Millard, Théorie générale du droit, Paris, Dalloz, 2006, p. 76 ; Hans Kelsen, Théorie générale des normes, O. Beaud (trad.), Paris, PUF, 1996, p. 344.

32. Hans Kelsen, Théorie pure du droit, op. cit., p. 256 et 286.

33. Jacques Derrida, Force de loi : le "fondement mystique de l'autorité», Paris, Galilée, 1994 (cité par Benoît Frydman, "Le droit, de la modernité à la postmodernité », Réseaux, $\mathrm{n}^{\circ} 88$, 2000, p. 69).

34. Konstantinos Stamastis, «La systématicité du droit chez Kelsen et les apories de la norme fondamentale», Arch. phil. droit 1986, p. 49. 
«idéalisme transcendantal ${ }^{35}$ », «résurgence de jusnaturalisme inconsciente $^{36}$ ", si ce n'est «sécularisation de l'idée de Dieu ${ }^{37}$ ", la norme fondamentale a été discutée et même dénoncée par nombre de grands penseurs du droit, y compris positivistes ${ }^{38}$. La norme fondamentale serait, dans l'œuvre kelsénienne, une source d'ambiguïté et d'apories internes bien plus qu'une source de logique et de scientificité ${ }^{39}$. Aussi a-t-elle pu se voir qualifiée de «coquecigrue la plus fameuse de toute la littérature philosophico-juridique » ou de « sorte de béquille ou prothèse qui soutient tout le système ${ }^{40}$ ».

Faut-il, à l'aune de ces sévères critiques, envisager la norme fondamentale tel un instrument inconséquent et donc envisager l'intention de définir la souveraineté à travers la norme fondamentale telle une intention inconséquente? En ces lignes, est argué que, dès lors que souveraineté et norme fondamentale se rejoignent, l'une et l'autre peuvent

35. Éric Maulin, «Positivisme», dans Dictionnaire de la culture juridique, op. cit., p. 1177.

36. Christian Atias, Philosophie du droit, Paris, PUF, 1999, p. 150.

37. François Ost et Michel van de Kerchove, «La référence à Dieu dans la théorie pure du droit de Kelsen », dans Qu'est-ce que Dieu?, Bruxelles, Publications des facultés universitaires Saint-Louis, 1985, p. 285.

38. Herbert Hart, en premier lieu, a opposé à Kelsen l'idée de «règle de reconnaissance», soit une forme de norme fondamentale empiriste, davantage en prise avec l'observation, avec les faits et avec la pratique. Voir Herbert Hart, Le concept de droit, M. van de Kerchove (trad.), Bruxelles, Publications des facultés universitaires Saint-Louis, 1994, p. 138 et suiv. Hart expliquait que «la règle de reconnaissance n'existe que sous la forme d'une pratique complexe, mais habituellement concordante, qui consiste dans le fait que les tribunaux, les fonctionnaires et les simples particuliers identifient le droit en se référant à certains critères ». " Son existence est une question de fait» (p. 138). Voir également Philippe Gérard, "L'idée de règle de reconnaissance: valeur, limites et incertitudes », RIEJ, $\mathrm{n}^{\circ} 65,2010, \mathrm{p} .65$ et suiv. Norberto Bobbio, pour sa part, jugeait que le constat de l'effectivité de la constitution devrait suffire à fonder sa validité. Et de dénoncer la norme fondamentale comme foyer d'une "grande controverse métaphysique, insoluble par principe " (Essais de théorie du droit, C. Agostini et M. Guéret (trad.), Paris, LGDJ, 1998, p. 135).

39. Konstantinos Stamastis, art. cité, p. 49 et suiv.

40. Paul Amselek, "Une fausse idée claire: la hiérarchie des normes juridiques", RRJ 2007, p. 566 et 567. Et d'autres commentateurs ont fait du postulat de la norme fondamentale une construction tautologique «condamn[ant] le système kelsénien, un peu comme un bateau qui ne pourrait jamais accoster, à tourner sur lui-même » (Laurent Habib, «L'impur objet de la science du droit », Droits, $n^{\circ}$ 11, 1990, p. 100), tandis que François Ost et Michel van de Kerchove ont montré combien «la tentative des plus conséquents des auteurs positivistes en vue de réaliser la clôture ou l'autofondation du système juridique s'avère nécessairement un échec» (De la pyramide au réseau? Pour une théorie dialectique du droit, Bruxelles, Publications des facultés universitaires Saint-Louis, 2002, p. 373). 
trouver un peu du sens et de la portée explicative qui leur font défaut lorsqu'elles prospèrent pour l'une, ou périclitent pour l'autre, chacune de son côté.

Et peut-être conviendrait-il de «sauver» la norme fondamentale en la comprenant de manière plus positive, soit comme un "pont» logique permettant aux univers du Sein et du Sollen de se retrouver implicitement, comme un outil intellectuel servant à juridiciser le fait originellement constitutif du système. La fiction de la norme fondamentale semble bien jouer ce rôle: traduire l'effectivité de l'ordre normatif en validité juridique. Il n'en demeure pas moins que, dans la théorie kelsénienne, la norme fondamentale est ce qui fonde ultimement le droit au droit de l'État ${ }^{41}$. Il est dès lors tentant de réunir norme fondamentale et souveraineté, car cette dernière peut aussi se comprendre comme étant ce qui fonde ultimement le droit au droit de l'État.

\section{La réunion proposée de la souveraineté et de la norme fondamentale}

De prime abord, la souveraineté et la norme fondamentale ne paraissent guère avoir vocation à se rejoindre. Il semble pourtant exister au moins quelques points communs entre la souveraineté, "principe des principes du droit public ${ }^{42}$ », et la norme fondamentale, norme des normes du droit. En ces pages, est soutenu que la souveraineté est une norme suprême fictive conférant le droit au droit à l'État. Et c'est parce que la souveraineté est la norme fondamentale qu'il n'est pas utile de rechercher quelque «droit au droit au droit». De cette façon, la souveraineté se retrouve au cœur de la théorie du droit en ce qu'elle renforce le critère de la validité et en ce qu'elle explique ou même justifie le lien indéfectible unissant juridicité et étaticité. Pour beaucoup d'auteurs, les sources étatiques seraient les seules sources de règles de droit. La souveraineté, telle qu'ici comprise, est un outil logique servant à justifier cette mainmise de l'État sur le droit.

Il faut développer plus avant la conception selon laquelle la souveraineté serait une donnée juridique (2.1) et, plus précisément, la norme

41. Kelsen expliquait, notamment, que « la norme fondamentale se borne à déléguer une autorité créatrice de normes». «[...] Elle ne contient rien d'autre que l'institution d'un fait créateur de normes, l'habilitation d'une autorité créatrice de normes " (Théorie pure du droit, op. cit., p. 260 et 263). Où Kelsen lui-même paraît confirmer que la norme fondamentale serait une norme habilitatrice, une norme attributive de compétence.

42. Olivier Beaud, La puissance de l'État, op. cit., p. 12. 
fondamentale de l'ordre juridique (2.2). Le premier des arguments est peut-être à rechercher directement dans les écrits de Kelsen. En effet, le plus illustre des théoriciens du droit du $\mathrm{xx}^{\mathrm{e}}$ siècle, père de l'idée de norme fondamentale, a pu considérer que, «dire que l'État est souverain, c'est dire que l'unité de ce système de règles que nous appelons l'ordre étatique repose en dernière analyse sur une règle que l'on suppose la première de toutes, la norme fondamentale, celle qu'il n'est pas besoin de justifier et de déduire ${ }^{43}$ ».

\subsection{La souveraineté comme donnée juridique}

A précédemment été soutenu que la souveraineté serait nécessairement étatique. Il faut également convenir, en sens inverse, que l'État est nécessairement souverain. Ensuite, est un autre problème le fait que la souveraineté se reconnaisse à l'étaticité alors qu'il est difficile de reconnaître l'étaticité grâce à la souveraineté, difficile d'observer la souveraineté sans avoir au préalable identifié l'État. En d'autres termes, il paraît impossible de caractériser la réalité de la souveraineté sans avoir au préalable caractérisé la réalité de l'État et il serait périlleux de faire de la souveraineté une condition de l'État: il y a souveraineté parce qu'il y a État et non État parce qu'il y a souveraineté, à l'identique de la personne physique qui possède des droits du seul fait qu'elle est, sans que le fait d'avoir ces droits conduise à la qualité de personne physique. La souveraineté est un attribut qui apparaît automatiquement, grâce à un effet juridique, dès l'instant où les conditions factuelles d'existence (ou « éléments constitutifs») de l'État sont réunies.

La souveraineté apparaît indispensable à l'État en même temps que propre à l'État: il n'existe pas plus d'État sans souveraineté que de souveraineté sans État. Ensuite, si la souveraineté ne peut se comprendre qu'étatiquement, il faut ajouter qu'elle ne peut se comprendre que juridiquement. Elle serait connue de l'État et seulement de l'État; et elle se définirait et s'exprimerait par le droit et uniquement par le droit. La souveraineté serait donc une donnée juridique, non quelque élément politique, factuel ou ressortissant autrement du non-droit. Elle serait la prérogative juridique discriminante de l'État. Est ainsi considéré que la souveraineté ne pourrait et ne devrait s'entendre que juridiquement, que sa terre d'élection serait le droit, le droit constitutionnel spécialement, et que partout ailleurs elle ne saurait être à sa place ${ }^{44}$.

43. Hans Kelsen, «Aperçu d'une théorie générale de l'État», RDP 1926, p. 577.

44. Arnaud Haquet, Le concept de souveraineté en droit constitutionnel français, Paris, PUF, 2004. À l'inverse, le sociologue Georges Gurvitch séparait la «souveraineté 
On observe que la souveraineté est susceptible d'être utilisée de deux manières différentes: soit à des fins stratégiques ou de légitimation (en tant que principe de légitimité servant à emporter la conviction et à justifier des décisions politiques), soit à des fins cognitives (en tant que principe logique servant à interpréter et à expliquer certains phénomènes ${ }^{45}$ ). Il semble que le second usage soit plus naturel que le premier et que la souveraineté soit nécessairement travestie lorsqu'elle est transportée dans le monde politicien, dans le monde journalistique ou dans tout autre monde polémique - ce qui est fréquemment le cas tant elle est perçue par beaucoup d'acteurs de la scène politico-médiatique comme un slogan ou un mot magique. Son univers originel paraît être celui de la philosophie politique et de la théorie de l'État ${ }^{46}$. Ici, il est proposé d'inscrire la souveraineté dans le cadre de la théorie juridique de l'État ou même dans celui de la théorie du droit, à la suite des professeurs qui la définissent telle une "puissance de $\operatorname{droit}^{47}$ ", tel « un pouvoir qui n'est pas supérieur en fait, mais seulement en droit $^{48}$ ». Nul doute qu'il existe dans les faits des institutions ou des groupements plus puissants que l'État, au moins à l'échelle de certains pays; cela ne saurait impacter la souveraineté conçue comme une suprématie juridique et non comme une suprématie factuelle ou une suprématie réelle.

Partant, il s'agit de plaider pour une souveraineté comprise dans un sens strict et cantonnée à la définition juridique de l'État. Loin de considérer qu'elle serait l'instrument d'une idéologie politique ou bien un «élément de pur fait ${ }^{49}$ ", il faudrait l'envisager en tant que donnée juridique objective indispensable à la compréhension de la structure juridique de l'État et de son particularisme ontologique. La souveraineté se présente ainsi telle la pierre angulaire de la définition de l'État, ce qui

politique», renvoyant au «monopole de la contrainte inconditionnée», et la "souveraineté juridique» (Éléments de sociologie juridique, Paris, Aubier, 1940, p. 205). De même, Maurice Hauriou opérait une distinction entre «la conception politique de la souveraineté "indépendance" et la conception juridique de la souveraineté "faisceau de droits régaliens" " (Maurice Hauriou, Précis élémentaire de droit constitutionnel, $2^{\mathrm{e}}$ éd., Paris, Librairie du Recueil Sirey, 1930, p. 17).

45. Sabine Saurugger, «Théoriser l'État dans l'Union européenne ou la souveraineté au concret ", Jus Politicum, $\mathrm{n}^{\circ}$ 8, 2012, p. 17.

46. Voir Olivier Beaud, «Souveraineté», dans Philippe Raynaud et Stéphane Rials (dir.), Dictionnaire de philosophie politique, Paris, PUF, 1996, p. 625 et suiv.

47. Denis Baranger, Droit constitutionnel, op. cit., p. 35.

48. François Hamon et Michel Troper, Droit constitutionnel, Paris, LGDJ, 2015, p. 28.

49. Bertrand Pauvert, Droit constitutionnel: 30 fiches de synthèse, Paris, Studyrama, 2004, p. 82. 
signifie que la pierre angulaire de la définition de l'État est juridique et, en conséquence, que l'État est principalement une réalité juridique.

Ces explications apportées, le sens de la souveraineté n'apparait qu'un peu moins obscur. En tant qu'élément juridique définitionnel de l'État, elle demeure susceptible de renvoyer à une foultitude d'éléments ${ }^{50}$. En ces pages, elle est décrite sous les traits de la norme fondamentale de l'ordre juridique, de telle sorte qu'elle appartiendrait autant à la théorie du droit qu'à la théorie de l'État - à moins qu'il faille considérer que, comme souveraineté et norme fondamentale ne feraient qu'une, théorie de l'État et théorie du droit ne feraient qu'une. Pareille conception ne manque pas de poser quelques difficultés. En premier lieu, d'aucuns se demanderont à bon escient comment la souveraineté peut être juridique en soi si le droit découle d'elle. Et Georges Burdeau d'avancer logiquement que la souveraineté serait une «notion exclusivement politique» puisque «sa qualité est de ne dépendre d'aucun ordre juridique prééta$\mathrm{bli}^{51} »$. Ce n'est pas autre chose que le problème de la validité de la norme fondamentale qui est ici en cause: comment la norme fondamentale peut-elle être valide en soi si la validité découle d'elle? Autant que la validité de la norme fondamentale ne saurait qu'être supposée, la juridicité de la souveraineté ne saurait qu'être supposée. Et, comme la règle de reconnaissance hartienne ne peut être consacrée qu'au départ de l'observation des faits, la souveraineté juridique ne peut être consacrée qu'au départ de l'observation des faits. Certes, le droit n'existe pas avant la souveraineté ni avant l'État; mais cela s'explique par le fait que la souveraineté est le niveau le plus élevé du droit. Il faut présumer que le droit dérive des faits et que les normes juridiques temporellement et substantiellement premières sont forcément des faits-droit ${ }^{52}$. Mais, s'il est interdit de lier faits et droit, alors il reste seulement permis de supposer la souveraineté juridique de l'État, sans autre forme de procès.

\subsection{La souveraineté comme norme fondamentale}

"Souveraineté » dérive du terme latin «superus» signifiant "supérieur». Cependant, la souveraineté paraît devoir se comprendre non pas comme quelque chose de supérieur, mais comme quelque chose

50. Voir, par exemple, Antonio Truyol y Serra, «Souveraineté », Arch. phil. droit 1990, p. 313 et suiv.

51. Georges Burdeau, L'État, op. cit., p. 65.

52. Jean-Jacques Rousseau définissait la souveraineté comme un pouvoir absolu émané du pacte social (Du contrat social, 1762, L. II, chap. 3 ) : le «contrat» social serait un fait à partir duquel émergerait le droit sous la forme de la souveraineté. 
de "suprême », d' "ultime» ou, dit autrement, de "plus supérieur que tout autre». Ensuite, reste à savoir quel est cet objet suprême ou ultime auquel renvoie le terme "souveraineté». En ces lignes, il est postulé que la souveraineté serait la norme suprême, la norme ultime de l'ordre juridique, une norme supposée à des fins logiques et de laquelle découlerait la juridicité de toutes les normes constitutives de l'ordre normatif étatique. Cette norme, qui serait une norme attributive ou habilitatrice, une norme secondaire au sens de Hart, une norme à portée purement formelle, pourrait être formulée en ces termes: «L'État crée et applique le droit». Partant, la souveraineté est conçue comme une métaprérogative juridique conférant le droit au droit à l'État, comme la prérogative juridique suprême qui renferme toutes les autres. Il s'agirait d'une règle de droit objectif attribuant à l'être étatique un droit subjectif consistant en une compétence juridique générale. À l'instar de toute personne physique ou morale, la personne étatique possède des droits fondamentaux; et le plus important d'entre eux serait la souveraineté ${ }^{53}$.

Comprise de la sorte, la souveraineté appartient à l'ordre des procédures et des compétences et il serait peu opérant de chercher à en fournir une définition matérielle. Elle autorise l'État à réguler les activités de sa population dans l'espace de son territoire en utilisant, de manière inconditionnée et unilatérale, le droit sous ses diverses formes. Le droit au droit ne pouvant avoir d'autre titulaire originaire que l'État, nulle personne non étatique n'a la possibilité d'édicter ou de valider des règles de droit ou d'édifier des institutions juridiques, à moins de bénéficier d'une habilitation étatique expresse. Une grande différence entre l'État et les organisations politiques non étatiques se situerait donc dans le caractère originaire de la structure juridique du premier. Les collectivités territoriales, par exemple, ne peuvent profiter que d'un droit au droit dérivé. L'État, lui, peut juridiquement tout, tout le temps, dans les dimensions géosociales que constituent son territoire et sa population. Matériellement, rien ne peut échapper par nature à la compétence de l'État qui jouit d'une "capacité générale avec blanc-seing» et est autorisé à saisir par le droit tout problème nouveau ${ }^{54}$. Mais cela ne signifie pas que l'État puisse factuellement tout, tout le temps, car la souveraineté n'implique pas automatiquement la puissance concrète, bien qu'elle contribue à la renforcer, notamment par des effets symboliques et psychologiques.

53. Florence Poirat, «La doctrine des "droits fondamentaux" de l'État», Droits, $\mathrm{n}^{\circ}{ }_{16}$, 1992, p. 89.

54. Herbert Krüger, Allgemeine Staatslehre, Stuttgart, Kohlhammer, 1966, p. 760 (cité par Olivier Beaud, La puissance de l'État, op. cit., p. 146). 
En définitive, Thomas Hobbes avait sans doute raison de qualifier la souveraineté d' " artifice ${ }^{55}$ "; comme toutes les constructions juridiques, elle n'est pas autre chose, même si elle emporte des conséquences tout à fait tangibles.

L'association de la souveraineté et de la norme fondamentale parait légitimée par les définitions de la souveraineté retenues par beaucoup d'auteurs, parmi lesquels quelques glorieux penseurs du droit et de l'État ${ }^{56}$. Pour autant, l'affirmation selon laquelle «la souveraineté n'est

55. Thomas Hobbes, Léviathan, 1651.

56. En effet, l'idée de norme fondamentale est proche lorsque Georges Gurvitch spécifie la souveraineté comme un «droit subjectif de commander " (Éléments de sociologie juridique, op. cit., p. 104), lorsque Julien Laferrière y voit un «pouvoir de droit originaire et suprême» (Manuel de droit constitutionnel, Paris, Domat Montchrestien, 1947, p. 359, cité par Jean-Claude Zarka, Introduction au droit constitutionnel, Paris, Ellipses, 2011, p. 11) ou lorsqu'Adhémar Esmein la caractérise en tant que «droit de commander à tous les citoyens " (Éléments de droit constitutionnel, Paris, Larose, 1896, p. 1, cité par Raymond Carré de Malberg, Contribution à la théorie générale de l'État..., op. cit., t. I, p. 71). Il en va pareillement lorsque Léon Duguit préfère parler de «droit de souveraineté » que de «souveraineté » et critique ce «droit de souveraineté " en ce qu'il serait une "proposition d'ordre métaphysique ", "imaginaire et ne repos[ant] sur rien de réel» (Traité de droit constitutionnel, op. cit., t. I, p. IX et t. II, p. 3). L'idée de norme fondamentale n'est également pas loin lorsqu'on conçoit la souveraineté en tant que "pouvoir exclusif de composer ou modifier l'ordre juridique » (Éric Maulin, «Souveraineté », art. cité, p. 1435), comme une notion «à la fois fondatrice, créatrice et justificatrice du pouvoir [juridique] » (Albert Rigaudière, "L'invention de la souveraineté », Pouvoirs, n ${ }^{\circ} 67,1993$, p. 5), en tant que "pouvoir qui n'émane pas de quelque pouvoir supérieur» (Alexandre Passerin d'Entrèves, La notion d'État, Paris, Recueil Sirey, 1969, p. 128), comme concrétisation juridique du fait que «l'État ne s'autorise que de lui-même et constitue sa propre référence » (Denys de Béchillon, Qu'est-ce qu'une règle de droit?, Paris, Odile Jacob, 1997, p. 105) ou bien en tant que «source d'où procède directement ou indirectement toute légitimité institutionnelle» («Souveraineté», dans Dictionnaire de la science politique et des institutions politiques, Paris, Armand Colin, 2015). Encore, Laband, Jellinek, Carré de Malberg ou Eisenmann côtoyaient, parfois avant l'heure, l'idée de norme fondamentale lorsqu'ils caractérisaient la souveraineté tel un «droit propre de domination" (Paul Laband, Droit public de l'Empire allemand, op. cit., t. I, p. 112, cité par Raymond Carré de Malberg, Contribution à la théorie générale de l'État..., op. cit., t. I, p. 151), telle une capacité de se déterminer soi-même» (Georges Jellinek, L'État moderne et son droit, $2^{e}$ partie: Théorie juridique de l'État (1911), Paris, Panthéon-Assas, 2005, p. 140), telle la « somme des droits de puissance active » (Raymond Carré de Malberg, Contribution à la théorie générale de l'État..., op. cit., t. I, p. 71) ou telle une « discrétionnalité totale (Charles Eisenmann, Cours de droit administratif, t. II, Paris, LGDJ, 1983, p. 672, cité par Jacques Baguenard, L'État: une aventure incertaine, op. cit., p. 64). Et n'est-ce pas la norme fondamentale qui est indirectement décrite par qui confère à la souveraineté le visage d'un "véritable droit naturel » (Éric Maulin, «Souveraineté», art. cité, p. 1435) ou par qui observe qu'elle « est une notion juridique unique en ce qu'elle ne se fonde pas, elle, sur une 
qu'une autre façon de désigner la norme fondamentale » ne se retrouve, semble-t-il, nulle part parmi la littérature jus théorique. Simplement, les auteurs d'un récent Dictionnaire de la science politique et des institutions politiques ont pu écrire, sans réfléchir plus longuement aux conséquences de pareille incise, que «la souveraineté, norme fondamentale (Grundnorm) au sens de Kelsen, apparaît comme un moyen de légitimer l'ensemble de la construction constitutionnelle ${ }^{57}$ ", tandis qu'un philosophe en est venu, lui aussi à l'occasion d'un ouvrage paru il y a peu, à concevoir, sans développer plus avant cette idée, que "la souveraineté est la norme fondamentale d'un État dont dérivent toutes les autres normes de droit inhérentes à l'ordre juridique de cet État - elle est la clef de voûte du système juridique, qu'elle hiérarchise et clôt $^{58} »$. L'image de la clef de voûte de l'ordre juridique, afin de figurer la souveraineté-norme fondamentale, apparaît ô combien bienvenue.

Il ressort des précédentes observations le sentiment que le rapprochement de la souveraineté et de la norme fondamentale a déjà été,

source de droit». «Elle produit du droit, mais n'en est pas issue. Cette apparente aporie trahit l'apparentement de la souveraineté avec un concept théologique» (François de Smet, Aux origines théologiques de la souveraineté, op. cit.) ? En outre, l'association de la souveraineté et de la norme fondamentale apparait sous-jacente à la théorie dite du "décisionnisme», développée par Carl Schmitt. Selon cette doctrine, le fondement du droit se situerait dans la volonté du souverain. Cette dernière serait nécessaire et suffisante à la juridicité : « La souveraineté [...] constitue la source de tout droit, c'est-à-dire de toutes les normes et de tous les ordres qui en découlent " (Carl Schmitt, Les trois types de pensée juridique, M. Kôller (trad.), Paris, PUF, 1995, p. 83, cité par Xavier Magnon, Théorie(s) du droit, Paris, Ellipses, 2008, p. 20). Aux yeux des tenants du décisionnisme, la souveraineté serait bien à la base de la validité du droit (Alain Sériaux, Le Droit. Une introduction, Paris, Ellipses, 1997, p. 273). Et Georges Burdeau confortait fortement la thèse de la souveraineté-norme fondamentale lorsqu'il expliquait que, suivant son «acception la plus courante », la souveraineté serait un «ensemble de prérogatives déterminées qui n'appartiennent à nul autre et dont la jouissance place celui qui en est investi au degré suprême de la domination ». "Le souverain, enseignait Burdeau, c'est celui qui décide quelle est l'idée de droit valable dans la collectivité» (L'État, op. cit., p. 64). Enfin, Michel Troper achève d'accréditer l'intuition d'une identité entre souveraineté et norme fondamentale à l'instant où il constate combien " on ne peut manquer d'être frappé par la ressemblance profonde entre la théorie de la souveraineté et la théorie de la norme fondamentale». Et de relever que « la doctrine de la souveraineté est apparue précisément à l'époque où le système juridique a commencé à présenter la structure particulière que nous décrivons comme une hiérarchie des normes » (Le droit et la nécessité, Paris, PUF, 2011).

57. Voir "Souveraineté», dans Dictionnaire de la science politique et des institutions politiques, op. cit.

58. François de Smet, Aux origines théologiques de la souveraineté, op. cit. 
par le passé, entrepris à de nombreuses reprises bien que souvent inconsciemment - et nécessairement inconsciemment pour ce qui est des théoriciens du droit et/ou de l'État qui ont écrit avant Kelsen. Il faut gager que la définition de la souveraineté en tant que norme fondamentale de l'ordre juridique n'est guère iconoclaste. Peut-être même s'agit-il de sa définition la plus habituelle, quoique cela soit largement sous-entendu et inexpliqué. Par ailleurs, il faut remarquer que nulle dénonciation de l'idée selon laquelle souveraineté et norme fondamentale seraient la même chose ou proches d'être la même chose n'a été trouvée. Cela tend à accréditer l'hypothèse d'une adhésion généralisée, mais involontaire à la souveraineté-norme fondamentale. L'un des enjeux des précédentes lignes était de mettre en évidence cette donnée doctrinale.

Il convient, à présent, de préciser plus concrètement ce en quoi consiste ce droit au droit que la souveraineté-norme fondamentale octroie à l'État. Dès lors que, eu égard aux polysémie et monosémie respectives de la souveraineté et de la norme fondamentale, il s'agit moins de définir la norme fondamentale à travers la souveraineté que de définir la souveraineté à travers la norme fondamentale, il faut surtout interroger les conséquences sous l'angle de la pensée de l'État de la compréhension de la souveraineté en tant que norme fondamentale ${ }^{59}$.

\section{Conclusion}

Depuis que Jean Bodin a écrit ses Six livres de la République, l'histoire et la logique du droit, et l'histoire et la logique de l'État n'ont cessé de toujours plus se rejoindre. L'évolution de la pensée juridique a mené à une nouvelle manière de se représenter le droit, dans laquelle «droit moderne» rime avec «État moderne». Ce mouvement a atteint son paroxysme avec la «théorie pure » kelsénienne, dans laquelle «État» et «droit » ne sont que deux façons différentes de désigner un seul et même phénomène. Or, si « État» et «droit » ne sont que deux façons différentes de désigner un seul et même phénomène, il est tentant de considérer que «souveraineté » et «norme fondamentale» ne seraient que deux façons différentes de désigner un seul et même objet, soit la norme suprême et ultime de l'ordre juridique, une norme supposée et non posée permettant de recouvrir du voile de la validité juridique l'ensemble des normes

59. Pour ce qui est de la pensée du droit, les problématiques que génère la souveraineténorme fondamentale sont identiques à celles qu'a pu par le passé générer la norme fondamentale conçue par Kelsen, l'une n'étant pas différente de l'autre; il suffit donc, en la matière, de renvoyer à l'œuvre de l'illustre théoricien du normativisme et aux forts et nombreux commentaires qu'elle a suscités sur ce point. 
appartenant à l'ordre normatif étatique. Cette norme suprême supposée cautionne le monopole étatique du droit parce qu'elle chapeaute l'ordre juridique dans son ensemble, le clôture, et que tout ce qui n'est pas rattaché à elle est sans autre forme de procès exclu de l'empire du droit.

Toutefois, on a de tout temps cherché à identifier, expliquer et justifier le droit de différentes façons. Et, aujourd'hui, il devient commun de considérer que le droit devrait se comprendre par le fait; la rupture humienne entre être et devoir-être est chaque jour un peu plus remise en cause, emportant dans le même mouvement la remise en cause de la souveraineté-norme fondamentale. Aussi les explications fournies par Carré de Malberg au début du $\mathrm{xx}^{\mathrm{e}}$ siècle peuvent désormais convaincre plus d'un juriste : «La Constitution originaire n'est, comme l'État auquel elle donne naissance, qu'un pur fait, réfractaire à toute qualification juridique. [...] C'est donc une erreur fondamentale que de vouloir, comme l'ont prétendu certains auteurs, trouver du droit à la source des États et de leurs Constitutions ${ }^{60}$.» Peut-être le positivisme de Carré de Malberg est-il plus positiviste que le positivisme de Kelsen, c'està-dire plus pragmatique et plus réaliste. Et cette origine factuelle de l'État et du droit n'est pas nécessairement incompatible avec la fiction de la souveraineté-norme fondamentale : cette dernière pourrait être conservée, afin de lui faire jouer son rôle symbolique de légitimation, tout en acceptant qu'avant elle et au-dessus d'elle il y a des faits, que des faits lui ont donné la vie et peuvent la lui reprendre. En somme, il semble que la souveraineté-norme fondamentale soit l'élément suprême de l'ordre du droit et que l'ordre du droit soit soumis à l'ordre du fait. Partant, peut-être serait-il judicieux de faire reposer la théorie juridique de l'État sur une théorie sociologique de l'État ou, du moins, d'associer l'une à l'autre ${ }^{61}$. L'idée de théorie générale de l'État retrouverait tout son sens.

La séparation des domaines du fait et du droit ne paraît cependant ni devoir ni pouvoir s'effacer dès lors qu'est étudié non pas l'apparition de l'État et du droit, mais l'état actuel de l'État et du droit. Les droits que l'État a sur sa population et sur son territoire peuvent ne pas aller de pair avec de parfaites maîtrises et dominations dans les faits, cela ne saurait impacter les conclusions tirées au terme d'une étude proprement juridique. Il importe de séparer la réalité juridique et la réalité sociofactuelle. La souveraineté-norme fondamentale appartenant au monde juridique, elle ne peut être touchée que par des phénomènes

60. Raymond Carré de Malberg, Contribution à la théorie générale de l'État..., op. cit., t. I, p. 66-67.

61. Voir Boris Barraud, L'État : entre fait et droit, Paris, L'Harmattan, 2015, p. 47 et suiv. 
appartenant eux aussi au monde juridique. Une règle de droit existe dans son ordre juridique indépendamment des faits; la validité est indifférente à l'effectivité. Si la souveraineté accorde un droit subjectif de commander, que ce droit ne produise de facto que peu d'effets ne change rien à sa présence au sommet de l'ordre juridique. En revanche, si l'État venait à disparaître parce que péricliteraient son territoire, sa population et/ou son gouvernement, alors la souveraineté s'éteindrait, comme les droits d'une personne s'effacent avec la mort de leur titulaire. La souveraineté-norme fondamentale ne saurait être diminuée par l'ignorance, la contestation ou la désobéissance puisqu'elle a une existence de droit et non une existence de fait; elle ne peut s'évanouir avant que l'État lui-même ait fini de décliner en raison de la désagrégation de ses fondations matérielles.

Par conséquent, la prolifération et le succès actuels des sources de normes privées para-étatiques ne sauraient malmener la souveraineté. Ces normes sont sans influence sur le monde du droit puisqu'elles n'en font pas partie, puisqu'elles sont des normes sociales non juridiques. Que des modes non étatiques de production et d'application de règles viennent concurrencer la régulation par l'État ne porte en aucunement atteinte à la souveraineté et au monopole étatique du droit qui en résulte. En revanche, cela peut être le signe d'une compétition entre plusieurs formes de régulation sociale.

Pareille approche suppose d'accepter les thèses de la validité-juridicité et de la hiérarchie des normes. Or ces thèses sont actuellement aux prises avec diverses théories du pluralisme juridique, qui conquièrent chaque jour de nouvelles parts de marché au sein de la pensée juridique collective. De plus en plus de scientifiques du droit voient dans les normes étatiques un genre de normes juridiques parmi d'autres. La juridicité ainsi détachée de l'étaticité et donc de la souveraineté, cette dernière ne saurait impliquer quelque droit au droit propre à l'État. Il faudrait donc la repenser ou l'abandonner, à moins que ce droit au droit ne soit plus précisément un droit au droit pur, un droit à la juridicité absolue, ce qui permettrait d'envisager l'existence d'un droit extraétatique impur, l'existence de normes privées à la juridicité imparfaite. La théorie kelsénienne serait alors, plus qu'une théorie pure du droit, une théorie pure du droit pur.

Docteur en droit

Laboratoire interdisciplinaire droit et mutations sociales Université d'Aix-Marseille 